\title{
Introduction to the Special Issue on Application-Specific Systems, Architectures and Processors
}

\author{
Frank Hannig ${ }^{1}$. Dirk Koch ${ }^{2}$
}

Received: 31 August 2021 / Revised: 31 August 2021 / Accepted: 31 August 2021

(c) The Author(s) 2021, corrected publication 2022

The International Conference on Application-specific Systems, Architectures and Processors (ASAP) has a long tradition in various hardware-related research topics, including computer arithmetic, application-specific instruction-set processors and accelerators, heterogeneous computing ranging from embedded systems to computing infrastructures, and reconfigurable computing. The 2020 edition of ASAP [1] was hosted by the Department of Computer Science at The University of Manchester, UK and took place as a virtual conference during July 6-7, 2020.

This special issue of Springer's Journal of Signal Processing Systems covers various facets of the topics mentioned afore. The special issue is based on extended contributions of selected top-level papers presented at ASAP 2020 [2]. From 118 submitted papers, 21 long papers had been presented at ASAP 2020, and after a careful peer-review process, four extended manuscripts were accepted for inclusion in this special issue. It is our pleasure to introduce these articles in the following briefly.

The initial two articles of this special issue deal with computer arithmetic.

The first article, "A Reconfigurable Posit Tensor Unit with Variable-Precision Arithmetic and Automatic Data Streaming" by Neves, Tomás, and Roma [3], deals with the acceleration of deep neural networks (DNNs). The authors propose a reconfigurable tensor unit that deploys an array of variable-precision vector multiply-accumulate units. The new vector unit is compared against existing SIMD units

Frank Hannig

frank.hannig@fau.de

Dirk Koch

dirk.koch@manchester.ac.uk

1 Department of Computer Science - Hardware/Software Co-Design, Friedrich-Alexander-Universität ErlangenNürnberg (FAU), Cauerstr. 11, 91058 Erlangen, Germany

2 School of Computer Science, The University of Manchester, Oxford Road, M14 9LP Manchester, UK concerning energy efficiency. For a 45nm ASIC implementation, the proposed unit provides an increased performance and energy efficiency over the existing state-of-the-art tensor and SIMD units that are present in off-the-shelf chips.

The second article, "Efficient Floating-Point Implementation of the Probit Function on FPGAs" by Joldes and Pasca [4], revisits floating-point implementations of probit, which is the quantile function associated with the standard normal distribution. More specifically, the authors utilize embedded floating-point DSP blocks of recent FPGA families. The proposed implementations outperform existing single-precision floating-point FPGA realizations in terms of area, latency, and accuracy.

The following two articles deal with heterogeneous accelerator systems.

The article with the title "How Many CPU Cores is an FPGA Worth? Lessons Learned from Accelerating String Sorting on a CPU-FPGA System," by Asiatici, Maiorano, and Ienne [5], presents a parallel hybrid superscalar string sample sorter on Intel HARPv2, a heterogeneous CPUFPGA system. The authors discuss the performance and energy advantages of FPGA-based systems in different data center settings. A dedicated aspect of this work is a sorting architecture for variable-length strings.

Vandebon, Coutinho, and Luk [6] provide the last article, "Scheduling Hardware-Accelerated Cloud Functions." The authors present a Function-as-a-Service (FaaS) approach for deploying managed cloud functions onto heterogeneous cloud infrastructures. The FaaS approach is validated for several applications stemming from machine learning, bioinformatics, and physics.

At the end of this editorial, we want to thank everybody who contributed to this special issue. We are very grateful to the editor-in-chief, Sun-Yuan Kung, and the co-editorsin-chief, Shuvra S. Bhattacharyya and Jarmo Takala, of Springer's Journal of Signal Processing Systems. We also acknowledge the administrative staff for their valuable support throughout the preparation and publication of this 
special issue. Furthermore, we thank all authors for their contributions to this special issue and their excellent efforts. Finally, we also thank all the reviewers for their careful work and valuable suggestions that helped improve the quality of the articles.

Funding Open Access funding enabled and organized by Projekt DEAL.

Open Access This article is licensed under a Creative Commons Attribution 4.0 International License, which permits use, sharing, adaptation, distribution and reproduction in any medium or format, as long as you give appropriate credit to the original author(s) and the source, provide a link to the Creative Commons licence, and indicate if changes were made. The images or other third party material in this article are included in the article's Creative Commons licence, unless indicated otherwise in a credit line to the material. If material is not included in the article's Creative Commons licence and your intended use is not permitted by statutory regulation or exceeds the permitted use, you will need to obtain permission directly from the copyright holder. To view a copy of this licence, visit http://creativecommons.org/licenses/by/4.0/.

\section{References}

1. Koch, D., Navaridas, J., \& Hannig, F. (2020, July). Message from the conference chairs - ASAP 2020. In Proceedings of the 31st
IEEE International Conference on Application-specific Systems, Architectures and Processors (ASAP) (pp. i-ii). IEEE. https://doi. org/10.1109/ASAP49362.2020.00005

2. Hannig, F., Navaridas, J., Koch, D., \& Abdelhadi, A. (Eds.) (2020, July). Proceedings of the 31st IEEE International Conference on Application-specific Systems, Architectures and Processors (ASAP), IEEE. ISBN: 978-1-7281-7147-0. https://doi.org/10. 1109/ASAP49362.2020

3. Neves, N., Tomás, P., \& Roma, N. (2021). A reconfigurable posit tensor unit with variable-precision arithmetic and automatic data streaming. Journal of Signal Processing Systems.

4. Joldes, M., \& Pasca, B. (2021) Efficient floating-point implementation of the Probit Function on FPGAs. Journal of Signal Processing Systems.

5. Asiatici, M., Maiorano, D., \& Ienne, P. (2021). How many CPU cores is an FPGA worth? Lessons learned from accelerating string sorting on a CPU-FPGA system. Journal of Signal Processing Systems.

6. Vandebon, J., Coutinho, J. G. F., \& Luk, W. (2021). Scheduling hardware-accelerated cloud functions. Journal of Signal Processing Systems.

Publisher's Note Springer Nature remains neutral with regard to jurisdictional claims in published maps and institutional affiliations. 